\title{
Overexpression of clusterin promotes angiogenesis via the vascular endothelial growth factor in primary ovarian cancer
}

\author{
YANXIA FU $^{1 *}$, YINGRONG LAI ${ }^{2 *}$, QIONGJUAN WANG ${ }^{1}$, XINGYANG LIU $^{1}$, WEIPENG HE ${ }^{1}$ \\ HAIHONG ZHANG ${ }^{3}$, CHUNYANG FAN ${ }^{3,4}$ and GUOFEN YANG ${ }^{1}$
}

\begin{abstract}
Departments of ${ }^{1}$ Gynecology and ${ }^{2}$ Pathology, The First Affiliated Hospital of Sun Yat-Sen University, Guangzhou, Guangdong 510080, P.R. China; ${ }^{3}$ Department of Pathology, University of Arkansas for Medical Sciences;

${ }^{4}$ Department of Pathology, Central Arkansas Veterans Healthcare System, Little Rock, AR 72205, USA
\end{abstract}

Received September 19, 2012; Accepted April 8, 2013

DOI: $10.3892 / \mathrm{mmr} .2013 .1436$

\begin{abstract}
Clusterin (CLU), a multifunctional glycoprotein, is ubiquitously produced in mammalian tissues. CLU has been shown to play significant roles in many of the biological behaviours of human tumors, such as cell proliferation, apoptosis, chemoresistance and angiogenesis. However, the relationship of CLU expression with angiogenesis in ovarian cancer has not been studied. A total of 275 epithelial ovarian tumors were obtained from archives of paraffin-embedded tissues. Immunohistochemical (IHC) staining for CLU and vascular endothelial growth factor (VEGF) was performed on a tissue microarray (TMA) including 181 primary ovarian epithelial cancer, 40 borderline ovarian tumors and 54 ovarian cancer mesenteric metastasis samples. Of the 174 cases, overexpression of CLU and VEGF were detected in 107 (61.5\%) and 109 (62.9\%) cases of primary ovarian carcinoma, respectively. Of the 107 cases of primary ovarian carcinoma with overexpression of CLU, expression of VEGF was increased in $82(75.2 \%)$ cases. However, in another 67 cases without CLU overexpression, overexpression of VEGF was observed in only $27(24.8 \%)$ cases $(\mathrm{P}<0.05)$. Overexpression of CLU in epithelial ovarian cancer appears to be correlated with increased tumor angiogenesis, consistent with the established role of CLU as an oncogene in the biology of ovarian cancer. In the treatment of ovarian cancer, these two markers may be used in the selection of patients for targeted therapy.
\end{abstract}

Correspondence to: Professor Guo-Fen Yang, Department of Gynecology, The First Affiliated Hospital of Sun Yat-Sen University, 58 Zhongshan Road II, Guangzhou, Guangdong 510080, P.R. China E-mail: pgf_yang@126.com

*Contributed equally

Key words: ovarian cancer, immunohistochemistry, clusterin, vascular endothelial growth factor

\section{Introduction}

Ovarian cancer is one of the three female genital malignant tumors. Its peak incidence is at $\geq 45$ years, and such genital malignancies have a high mortality rate. Despite advances in the treatment of ovarian cancer, the incidence of ovarian cancer is on the increase in Asian countries such as China and Singapore. Recent cancer statistics have demonstrated the 5 -year survival rate of ovarian cancer to be $\sim 44 \%$ (1). If the tumor is found when it is localized, the 5-year survival rate is $\sim 92 \%$. However, $\sim 70 \%$ of patients diagnosed with ovarian cancer at an advanced stage (FIGO III/IV), have a 5 -year survival rate of only $27 \%$ (1). It is well known that epithelial ovarian cancer is the most common pathological type of ovarian cancer. Moreover, the progression of ovarian cancer is considered to be the complex pathophysiology of a number of genes to change the multistep process. Therefore, the molecular carcinogenic mechanisms of closely related genes in ovarian cancer and their clinical significance, as well as early diagnosis using ovarian cancer molecular markers and new therapeutic targets, is the key to improving the life of patients with ovarian cancer.

In a previous study, clusterin (CLU) was identified as indicator that can be used to determine the prognosis of ovarian cancer (2). Human CLU gene is located on chromosome $8 \mathrm{p} 21$, contains a glycoprotein composed of 1,651 bp encoding 448 amino acids. CLU protein, cleaved from a cytoplasmic protein, was originally derived from rat testis fluid. Due to its phytohemagglutinin-like properties, it promotes suspension cell aggregation known as cluster agglutinin (CLU). Subsequently, CLU is widely expressed in human and mammalian tissues and secreted in many human body fluids. CLU has numerous biological functions, such as anti-apoptosis, cell cycle regulation, DNA repair, cell adhesion, tissue remodeling, lipid reconstruction and transfer, membrane reuse, as well as oxidative stress and aging (3). Previous studies confirmed that, many physiological functions of CLU are important in the development of certain human malignancies, such as hepatocellular carcinoma, ovarian cancer, colon cancer, bladder cancer, breast cancer, and non-small cell lung cancer (4-9).

In 1983, Senger et al (10) identified a factor in the guinea pig tumor cell lines, designated as VPF, and confirmed that 
this factor was able to promote tumor angiogenesis for the first time (10). VPF was demonstrated to have a specific promotion angiogenesis effect, termed vascular endothelial growth factor (VEGF) (11). VEGF, in normal physiological conditions, is crucial in the maintenance of organ function. As with normal ovarian tissue, VEGF is involved in maintaining ovarian cyclical endocrine function (12). In the malignant tumors of a variety of solid tissues, VEGF was involved in angiogenesis, tumor development and invasion (13). In ovarian cancer, it has been previously shown that VEGF can produce normal and functional ovarian surface epithelial cells into the ascites inducing tumor cells (13). Ovarian cancer cells in vitro and in vivo can generate a large number of VEGF. VEGF has been considered a serological marker for ovarian cancer and for the prognosis thereof (12). Expression of VEGF in ovarian cancer is therefore associated with a number of growth factors, such as hypoxia-inducible factor 1- $\alpha$, placental grow th factor, tumor necrosis factor- $\alpha$ (TNF- $\alpha$ ), matrix metalloproteinases and lysophosphatidic acid (14-19). However, the relationship between the expression of CLU and VEGF in ovarian cancer has not been reported.

The main purpose of this study was to examine CLU and VEGF expression in epithelial ovarian cancer and to analyze their possible relationships in ovarian cancer progression. In addition, we aimed to understand the role of CLU in the progression of ovarian cancer.

\section{Materials and methods}

Patients and tissue specimens. In this study, a total of 275 epithelial ovarian tumors (including 181 primary ovarian epithelial cancer, 40 borderline ovarian tumors and 54 ovarian cancer mesenteric metastasis samples) were obtained from archives of paraffin-embedded tissues between 1996 and 2008 at the Department of Pathology, Cancer Center and the First Affiliated Hospital, Sun Yat-Sen University (Guangzhou, China). These 181 tumors consisted of 119 serous carcinoma, 23 mucinous carcinomas and 39 other types of carcinoma (endometroid, clear cell and undifferentiated). None of the patients underwent any chemotherapy prior to the surgery. The cancer cases selected were based on availability of resection tissue and follow-up data. The clinical and pathological data are provided in Table I. For the use of these clinical materials for research purposes, prior patient consent as well as approval from the Institute Research Medical Ethics Committee of Sun Yat-Sen University were obtained.

Tissue microarray (TMA). TMAs were constructed as described in a previous study (20). Tissue HE staining was observed under the microscope slides, and typical lesions (cancer-rich) were identified, according to observations of the 'donor' paraffin-embedded tissue corresponding to the typical marked lesions, while preparing for an unorganized blank wax block as a 'recipient' paraffin block. Then, using a tissue microarray production machine (Beecher Instruments, Silver Spring, MD, USA), $0.6 \mathrm{~mm}$ diameter cylindrical desired target tissue was removed from the 'donor' paraffin-embedded tissue specimens, and a preset 'receptor' was inserted within the hollow wax block. The procedure was repeated, and specimens were arranged in a micro-tissue array, made of paraffin TMA. Multiple sections $(5 \mu \mathrm{m})$ were cut from the TMA block and mounted on microscope slides. In order to ensure the validity of TMA, three cores of the samples were selected from each tumor tissue.

Immunohistochemical staining. Immunohistochemical (IHC) staining for CLU and VEGF was performed on an ovarian tumor TMA. Mouse anti-human clusterin monoclonal antibody (Clone 41D, in Upstate Biotechnology; $1 / 100$ dilutions) was used for antigen retrieval. As the negative control, 10 cases of normal ovarian tissue were immunohistochemically stained using the same antibody and staining procedure. A staining index (0-9) was obtained for each sample by multiplying the staining intensity (0-3: 0 , negative; 1 , weak; 2 , moderate and 3 , strong) with percentages of positively stained cells $(1-3: 1, \leq 10 \% ; 2,10-50 \%$ and $3, \geq 50 \%$ ). Protein expression with a scoring index of $\geq 4$ was considered to be overexpressed. Use of the staining index of 4 as the cut-off to divide between positive and negative CLU IHC staining results was based on the CLU IHC staining results of 10 normal ovarian samples, the staining indices of which ranged from 0 to 4 .

For VEGF, assessment of the results was in accordance with the staining intensity and percentage of positive cells. According to the same scoring methods as CLU, the protein expression with a scoring index of $\geq 4$ was considered to be overexpressed. Observations were recorded and assessed by two independent pathologists, who were blind to the identity of the samples.

Statistical analysis. Statistical analysis was performed using the SPSS statistical software package (SPSS Standard version 13.0, SPSS Inc., Chicago, IL, USA). The test was used to evaluate differences in CLU and VEGF expression between two categories of tissues. The association between variables was assessed by the Chi-square analysis. In all cases, differences with a $\mathrm{P}<0.05$ were considered to indicate a statistically significant difference.

\section{Results}

CLU expression in ovarian tissues and metastasis tissues. CLU expression could be evaluated informatively in $174 / 181$ of ovarian carcinomas, 37/40 of borderline tumors and 53/54 of metastasis tissues in TMA tissues. The non-informative TMA samples included unrepresentative samples, samples with insufficient tumor cells ( $<300$ cells per case) and lost samples. According to the abovementioned definition, the overexpression of CLU was detected in 107/174 (61\%) primary ovarian carcinomas. The decreasing frequencies of CLU expression in primary ovarian carcinomas (61\%), in borderline tumors $(62 \%)$ and in metastasis $(37 \%)$ indicated a significant difference $(\mathrm{P}<0.05$, Table II). IHC staining of CLU protein is shown in Fig. 1A-D.

Association of CLU expression with ovarian carcinoma and patient clinicopathological characteristics. The association between CLU expression in ovarian carcinomas and several 
Table I. Clinicopathological parameters for the prognosis of 174 patients with invasive ovarian carcinoma by univariate survival analysis (log-rank test).

\begin{tabular}{|c|c|c|c|c|}
\hline Variable & All cases & $\begin{array}{l}\text { Mean survival } \\
\text { (months) }\end{array}$ & $\begin{array}{l}\text { Median survival } \\
\text { (months) }\end{array}$ & P-value \\
\hline Age at surgery (years) & & & & 0.889 \\
\hline$\leq 50^{\mathrm{a}}$ & 89 & 80.4 & 66.0 & \\
\hline$>50$ & 85 & 81.4 & 62.0 & \\
\hline Histological type & & & & 0.757 \\
\hline Serous & 115 & 76.1 & 62.0 & \\
\hline Mucinous & 22 & 79.8 & $\mathrm{NR}^{\mathrm{b}}$ & \\
\hline Others $^{c}$ & 37 & 97.1 & NR & \\
\hline Histological grade (Silveberg) & & & & 0.015 \\
\hline G1 & 31 & 108.0 & 136.0 & \\
\hline $\mathrm{G} 2$ & 102 & 74.9 & 64.0 & \\
\hline G3 & 41 & 49.1 & 29.0 & \\
\hline pT status & & & & 0.001 \\
\hline pT1 & 48 & 114.2 & NR & \\
\hline pT2 & 34 & 80.4 & NR & \\
\hline pT3 & 92 & 63.7 & 35.0 & \\
\hline $\mathrm{pN}$ status & & & & $<0.001$ \\
\hline pNO & 86 & 92.8 & 136.0 & \\
\hline $\mathrm{pN} 1$ & 88 & 55.9 & 39.0 & \\
\hline pM status & & & & $<0.001$ \\
\hline $\mathrm{pMX}$ & 150 & 89.6 & 136.0 & \\
\hline pM1 & 24 & 23.0 & 13.0 & \\
\hline FIGO stage & & & & $<0.001$ \\
\hline I & 33 & 130.4 & NR & \\
\hline II & 20 & 113.7 & NR & \\
\hline III & 97 & 69.0 & 39.0 & \\
\hline IV & 24 & 23.0 & 13.0 & \\
\hline
\end{tabular}

${ }^{a}$ Mean age. ${ }^{b}$ Not reached. ${ }^{c}$ Endometrioid, clear cell and undifferentiated types.

Table II. The expression of CLU in epithelial ovarian carcinomas, borderline ovarian tumors and metastasis.

\begin{tabular}{lccc}
\hline & & & CLU protein expression \\
\cline { 3 - 4 } Item & All cases & $(-), \mathrm{n}(\%)$ & $(+), \mathrm{n}(\%)$ \\
\hline Tissue & & & 0.015 \\
Epithelial ovarian carcinomas & 174 & $67(39)$ & $107(61)$ \\
Borderline ovarian tumors & 37 & $14(38)$ & $23(62)$ \\
Metastasis & 53 & $32(60)$ & $21(40)$ \\
\hline
\end{tabular}

${ }^{\mathrm{a} C h i}$-square test. CLU, clusterin.

known clinicopathological characteristics was investigated. CLU expression was positively correlated with histological grade and type $(\mathrm{P}<0.05$, Table III). No significant correlation was obtained between CLU expression and FIGO stage, $\mathrm{pT} / \mathrm{pN} / \mathrm{pM}$ status, patient age ( $\mathrm{P}>0.05$, Table III).
VEGF expression in ovarian tissues and metastasis tissues. VEGF expression could be evaluated informatively in TMA tissues of 174/181 of ovarian carcinomas, 36/40 of borderline tumors and 53/54 of metastasis tissues. Overexpression of VEGF was detected in 109/174 (63\%) of primary ovarian carci- 


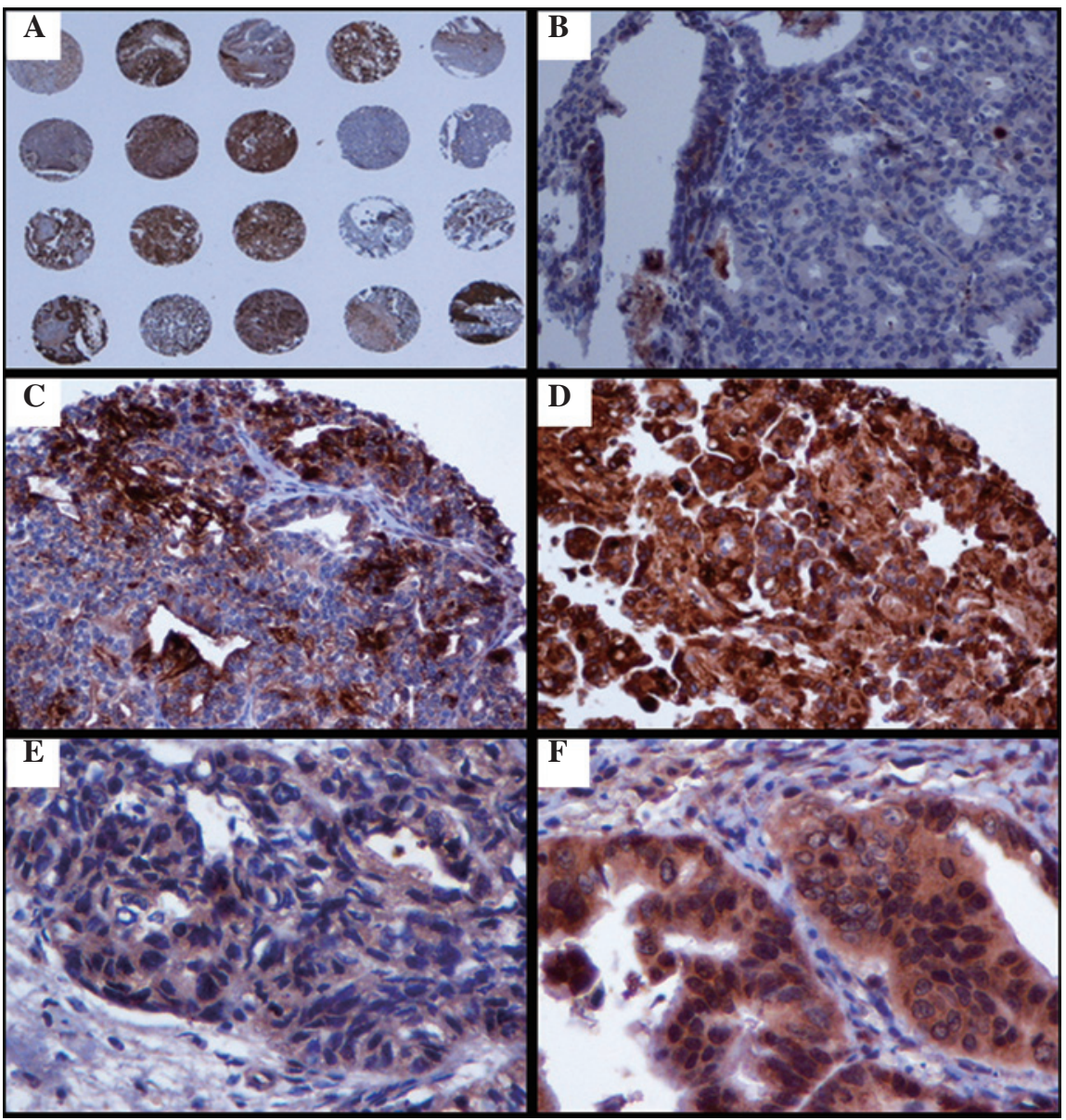

Figure 1. Immunohistochemical (IHC) staining of clusterin (CLU) and vascular endothelial growth factor (VEGF) in human ovarian tissues. Examples of negative clusterin expression [(B) staining index of 0], moderate clusterin expression [(C) staining index of 6] and high clusterin expression [(D) staining index of 9] are shown. Magnifications: (A) x40; (B-D) x200. (E) Negative expression of VEGF was observed in a normal surface epithelium of ovary (magnification, $\mathrm{x} 400$ ). (F) Overexpression of VEGF was detected in an ovarian carcinoma (magnification, $\mathrm{x} 400$ ).

nomas. In borderline ovarian tumors and metastasis tissues, the VEGF expression rate was $19 \%(7 / 37)$ and $94 \%(50 / 53)$ respectively. The difference of expression levels was statistically significant $(\mathrm{P}<0.05$, Table IV). Immunohistochemical staining of VEGF is shown in Fig. 1E-F.

Association of VEGF expression with ovarian carcinoma patient clinicopathological characteristics. The association between VEGF expression in ovarian carcinomas and several known clinicopathological characteristics was examined. VEGF expression was positively correlated with histological type and grade $(\mathrm{P}<0.05$, Table $\mathrm{V})$. No significant correlation was observed between VEGF expression and $\mathrm{pT} / \mathrm{pN} / \mathrm{pM}$ status, FIGO stage and patient age $(\mathrm{P}>0.05$, Table V).

Association between the expression of CLU and VEGF in ovarian cancer tissues. Protein overexpression of CLU and VEGF was detected in 107 (61\%) and 109 (63\%) cases, respectively. However, in 107 cases of CLU protein overexpression, the rate of overexpression and negative expression of VEGF were $82(77 \%)$ and $25(23 \%)$ cases, respectively, reflecting a significant increase of CLU protein expression and an increase in VEGF expression in ovarian cacinoma. The Chi-square test revealed a positive correlation between VEGF and CLU expression $(\mathrm{r}=0.343, \mathrm{P}<0.05$, Table VI).

\section{Discussion}

In recent years, there has been a marked improvement in the treatment of ovarian cancer, although the mortality rate of ovarian cancer remains high. Recent statistics show that the 5 -year survival rate of ovarian cancer is $44 \%$, whereas for advanced stage, the 5-year survival rate is $<30 \%$ (1). The lack of available biological markers for accurate prediction of clinicopathological parameters and prognosis largely contribute to the high mortality rate of ovarian cancer. Early cancer detection and the lack of novel therapeutic targets may yield new strategies for the treatment of ovarian cancer. The incidence of occult ovarian tumors is difficult to be diagnosed at an early stage as no symptoms are evident at this stage. Thus, the prognosis for the majority of patients diagnosed with ovarian cancer at an advanced stage, is poor. Therefore, the identification of novel genetic biomarkers is crucial as this would allow the early detection of cancer, provide new therapeutic targets for cancer treatments and ultimately improve survival for patients with ovarian carcinoma.

CLU has numerous biological functions, such as anti-apoptosis, cell cycle regulation, DNA repair, cell adhesion, tissue remodeling, lipid reconstruction and transfer, the membrane reuse, as well as oxidative stress and aging (3). In a recent study, it was concluded that CLU is a potential molecular 
Table III. Association of CLU expression with patient clinicopathological characteristics in epithelial ovarian carcinomas.

\begin{tabular}{|c|c|c|c|c|}
\hline \multirow[b]{2}{*}{ Variable } & \multirow[b]{2}{*}{ All cases } & \multicolumn{3}{|c|}{ CLU protein expression } \\
\hline & & $(-), \mathrm{n}(\%)$ & $(+), \mathrm{n}(\%)$ & $\mathrm{P}$ value $^{\mathrm{a}}$ \\
\hline Age at surgery (years) & & & & 0.692 \\
\hline$\leq 50.7$ & 89 & $33(37)$ & $56(63)$ & \\
\hline$>50.7$ & 85 & $34(40)$ & $51(60)$ & \\
\hline Histological type & & & & 0.002 \\
\hline Serous & 115 & $35(30)$ & $80(70)$ & \\
\hline Mucinous & 22 & $15(68)$ & $7(32)$ & \\
\hline Others & 37 & $17(46)$ & $20(54)$ & \\
\hline Histological grade (Silverberg) & & & & 0.004 \\
\hline G1 & 31 & $20(65)$ & $11(35)$ & \\
\hline G2 & 102 & $34(33)$ & $68(67)$ & \\
\hline G3 & 41 & $13(32)$ & $28(68)$ & \\
\hline pT status & & & & 0.471 \\
\hline pT1 & 48 & $20(42)$ & $28(58)$ & \\
\hline pT2 & 34 & $10(29)$ & $24(71)$ & \\
\hline pT3 & 92 & $37(40)$ & $55(60)$ & \\
\hline pN status & & & & 0.557 \\
\hline pNO & 86 & $35(41)$ & $51(59)$ & \\
\hline $\mathrm{pN} 1$ & 88 & $32(36)$ & $56(64)$ & \\
\hline pM status & & & & 0.575 \\
\hline $\mathrm{pMX}$ & 150 & 59 (39) & $91(61)$ & \\
\hline pM1 & 24 & $8(33)$ & $16(67)$ & \\
\hline FIGO stage & & & & 0.243 \\
\hline I & 33 & $17(52)$ & $16(48)$ & \\
\hline II & 20 & $5(25)$ & $15(75)$ & \\
\hline III & 97 & $37(38)$ & $60(62)$ & \\
\hline IV & 24 & $8(33)$ & $16(67)$ & \\
\hline
\end{tabular}

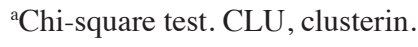

Table IV. VEGF expression in epithelial ovarian carcinomas, borderline ovarian tumors and metastasis.

\begin{tabular}{lcrr}
\hline & & & VEGF \\
\cline { 3 - 4 } Item & All cases & $(-), \mathrm{n}(\%)$ & $(+), \mathrm{n}(\%)$ \\
\hline Tissue & & & \multicolumn{2}{c}{ P-value ${ }^{\mathrm{a}}$} \\
Epithelial ovarian carcinomas & 174 & $65(37)$ & $109(63)$ \\
Borderline ovarian tumors & 36 & $28(78)$ & $8(22)$ \\
Metastasis & 53 & $2(4)$ & $51(96)$ \\
\hline
\end{tabular}

${ }^{\mathrm{a} C h i}$-square test. VEGF, vascular endothelial growth factor.

target for predicting survival, while the targeting of this s-CLU may improve survival of patients with ovarian cancer (21). We previously reported that the overexpression of CLU protein was correlated with more aggressive clinical behavior in ovarian cancer (2). However, no studies have confirmed the carcinogenic mechanism of the CLU gene in ovarian cancer. This study aimed to dectect CLU gene and VEGF expression in ovarian cancer and to analyze the association and possible carcinogenic mechanism of CLU in ovarian cancer. In our study, CLU expression could be evaluated informatively in 
Table V. Association of VEGF expression with patient clinicopathological characteristics in epithelial ovarian carcinomas.

\begin{tabular}{|c|c|c|c|c|}
\hline \multirow[b]{2}{*}{ Variable } & \multirow[b]{2}{*}{ All cases } & \multicolumn{3}{|c|}{ VEGF } \\
\hline & & $(-), \mathrm{n}(\%)$ & $(+), \mathrm{n}(\%)$ & P-value ${ }^{a}$ \\
\hline Age at surgery (years) & & & & 0.696 \\
\hline$\leq 50.7$ & 89 & $32(36)$ & $57(64)$ & \\
\hline$>50.7$ & 85 & $33(39)$ & $52(61)$ & \\
\hline Histological type & & & & 0.146 \\
\hline Serous & 115 & $38(33)$ & $77(67)$ & \\
\hline Mucinous & 22 & $12(55)$ & $10(45)$ & \\
\hline Others & 37 & $15(41)$ & $22(59)$ & \\
\hline Histological grade (Silveberg) & & & & 0.031 \\
\hline G1 & 31 & $18(58)$ & $13(42)$ & \\
\hline G2 & 102 & $34(33)$ & $68(67)$ & \\
\hline G3 & 41 & $13(32)$ & $28(68)$ & \\
\hline pT status & & & & 0.112 \\
\hline pT1 & 48 & $23(48)$ & $25(52)$ & \\
\hline $\mathrm{pT} 2$ & 34 & $14(41)$ & $20(59)$ & \\
\hline pT3 & 92 & $28(30)$ & $64(70)$ & \\
\hline $\mathrm{pN}$ status & & & & 0.066 \\
\hline $\mathrm{pN} 0$ & 86 & $38(44)$ & $48(56)$ & \\
\hline $\mathrm{pN} 1$ & 88 & $27(31)$ & $61(69)$ & \\
\hline pM status & & & & 0.372 \\
\hline $\mathrm{pMX}$ & 150 & $58(39)$ & $92(61)$ & \\
\hline pM1 & 24 & $7(29)$ & $17(71)$ & \\
\hline FIGO stage & & & & 0.087 \\
\hline I & 33 & $18(55)$ & $15(45)$ & \\
\hline II & 20 & $9(45)$ & $11(55)$ & \\
\hline III & 97 & $31(32)$ & $65(68)$ & \\
\hline IV & 24 & $7(29)$ & $17(71)$ & \\
\hline
\end{tabular}

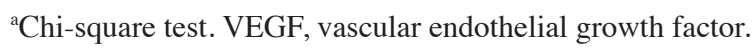

Table VI. Association between the expression of clusterin (CLU) and VEGF in ovarian cancer tissues.

\section{CLU protein expression}

\begin{tabular}{llll}
\cline { 2 - 4 } VEGF protein expression & $(-)$ & $(+)$ & P-value \\
\hline$(-)$ & 40 & 25 & $<0.001$ \\
$(+)$ & 27 & 82 &
\end{tabular}

Chi-square test, 23.247. r=0.343. VEGF, vascular endothelial growth factor.

174/181 of ovarian carcinomas, 37/40 of borderline tumors and 53/54 of metastasis tissues in TMA tissues. The decreasing frequencies of CLU expression in primary ovarian carcinomas $(61 \%)$, in borderline tumors $(57 \%)$ and in metastasis $(37 \%)$ exhibited a significant difference $(\mathrm{P}<0.05)$.

In ovarian cancer, it was previously shown that VEGF is capable of producing normal and functional ovarian surface epithelial cells into ascites inducing tumor cells (13). Ovarian cancer cells in vitro and in vivo can generate a large number of VEGF. VEGF has been considered to be used as a serological marker for ovarian cancer as well as for the prognosis of ovarian cancer (12). It has been confirmed that a high expression of VEGF in ovarian cancer occurs due to lack of oxygen and a variety of growth factors including insulin-like growth factor-1 and EGF and platelet-derived growth factor, transforming growth factor- $\beta$ and TNF- $\alpha$ in ovarian cancer have been proven to promote VEGF expression (18). In ovarian cancer, the association of VEGF with CLU expression has not been reported. In the present study, in the 107 cases of CLU protein overexpression, the rate of overexpression and negative expression of VEGF were $82(77 \%)$ and $25(23 \%)$ cases, respectively, reflecting a significant increase of CLU protein expression and an increase in VEGF expression in ovarian carcinoma. Further analysis confirmed that a positive correlation was observed between VEGF and CLU expression. CLU overexpression may promote the expression of VEGF leading to angiogenesis in ovarian cancer. The above findings provide further evidence for CLU as an oncogene in the biology of ovarian cancer. 
VEGF is involved in the progression of a variety of malignant tumors, leading to a poor prognosis. The use of bevacizumab in clinical practice for drug-resistant ovarian cancer patients has been beneficial (22). However, due to its high cost, bevacizumab is used only as a second-line treatment for malignant tumors. Treatments often fail to achieve satisfactory results when tumor cells are multi-drug resistant. The present study has demonstrated the close correlation between CLU and VEGF expression in primary ovarian cancer. In clinical practice, in order to obtain higher efficiency and to reduce adverse drug reactions and treatment costs, the combined use of CLU and VEGF may be applied to the selection of patients for bevacizumab treatment.

In conclusion, overexpression of CLU in epithelial ovarian cancer appears to be correlated with increased tumor angiogenesis, which is consistent with the established role of CLU as an oncogene in the biology of ovarian cancer. In the treatment of ovarian cancer, these two markers may be useful in the selection of patients for targeted therapy.

\section{Acknowledgements}

This study was support by the Nature Science Foundation of China (no. 81272855) and Project of Guangdong Science and Technology (no. 2010B060900098).

\section{References}

1. Siegel R, Naishadham D and Jemal A: Cancer statistics. CA Cancer J Clin 62: 10-29, 2012.

2. Yang GF, Li XM and Xie D: Overexpression of clusterin in ovarian cancer is correlated with impaired survival. Int J Gynecol Cancer 19: 1342-1346, 2009.

3. Trougakos IP and Gonos ES: Clusterin/apolipoprotein J in human aging and cancer. Int J Biochem Cell Biol 34: 1430-1448, 2002.

4. Rizzi F and Bettuzzi S: Clusterin (CLU) and prostate cancer. Adv Cancer Res 105: 1-19, 2009.

5. Albert JM, Gonzalez A, Massion PP, Chen H, Olson SJ, Shyr Y, Diaz R, Lambright ES, Sandler A, Carbone DP, Putnam JJ, Johnson DH and Lu B: Cytoplasmic clusterin expression is associated with longer survival in patients with resected non small cell lung cancer. Cancer Epidemiol Biomarkers Prev 16 : 1845-1851, 2007.

6. Pucci S, Bonanno E, Pichiorri F, Angeloni C and Spagnoli LG: Modulation of different clusterin isoforms in human colon tumorigenesis. Oncogene 23: 2298-2304, 2004.

7. Redondo M, Villar E, Torres-Munoz J, Tellez T, Morell M and Petito CK: Overexpression of clusterin in human breast carcinoma. Am J Pathol 157: 393-399, 2000.
8. Miyake H, Gleave M, Kamidono S and Hara I: Overexpression of clusterin in transitional cell carcinoma of the bladder is related to disease progression and recurrence. Urology 59: 150-154, 2002.

9. Lau SH, Sham JS, Xie D, Tzang CH, Tang D, Ma N, Hu L, Wang Y, Wen JM, Xiao G, Zhang WM, Lau GK, Yang M and Guan XY: Clusterin plays an important role in hepatocellular carcinoma metastasis. Oncogene 25: 1242-1250, 2006.

10. Senger DR, Galli SJ, Dvorak AM, Perruzzi CA, Harvey VS and Dvorak HF: Tumor cells secrete a vascular permeability factor that promotes accumulation of ascites fluid. Science 219: 983-985, 1983.

11. Connolly DT, Olander JV, Heuvelman D, Nelson R, Monsell R, Siegel N, Haymore BL, Leimgruber R and Feder J: Human vascular permeability factor. Isolation from U937 cells. J Biol Chem 264: 20017-20024, 1989.

12. Geva E and Jaffe RB: Role of vascular endothelial growth factor in ovarian physiology and pathology. Fertil Steril 74: 429-438, 2000.

13. Ramakrishnan S, Subramanian IV, Yokoyama Y and Geller M: Angiogenesis in normal and neoplastic ovaries. Angiogenesis 8: 169-182, 2005.

14. Belotti D, Paganoni P, Manenti L, Garofalo A, Marchini S, Taraboletti G and Giavazzi R: Matrix metalloproteinases (MMP9 and MMP2) induce the release of vascular endothelial growth factor (VEGF) by ovarian carcinoma cells: implications for ascites formation. Cancer Res 63: 5224-5229, 2003.

15. Matei D, Kelich S, Cao L, Menning N, Emerson RE, Rao J, Jeng MH and Sledge GW: PDGF BB induces VEGF secretion in ovarian cancer. Cancer Biol Ther 6: 1951-1959, 2007.

16. Liao S, Liu J, Lin P, Shi T, Jain RK and Xu L: TGF-beta blockade controls ascites by preventing abnormalization of lymphatic vessels in orthotopic human ovarian carcinoma models. Clin Cancer Res 17: 1415-1424, 2011.

17. Cao Z, Fang J, Xia C, Shi X and Jiang BH: trans-3,4,5'-Trihydroxystibene inhibits hypoxia-inducible factor $1 \alpha$ and vascular endothelial growth factor expression in human ovarian cancer cells. Clin Cancer Res 10: 5253-5263, 2004.

18. Masoumi MS, Amini A, Morris DL and Pourgholami MH: Significance of vascular endothelial growth factor in growth and peritoneal dissemination of ovarian cancer. Cancer Metastasis Rev 31: 143-162, 2012.

19. Liu LZ, Hu XW, Xia C, He J, Zhou Q, Shi X, Fang J and Jiang BH: Reactive oxygen species regulate epidermal growth factor-induced vascular endothelial growth factor and hypoxia-inducible factor-1alpha expression through activation of AKT and P70S6K1 in human ovarian cancer cells. Free Radic Biol Med 41: 1521-1533, 2006.

20. Jones PA and Baylin SB: The fundamental role of epigenetic events in cancer. Nat Rev Genet 3: 415-428, 2002.

21. Hassan MK, Watari H, Han Y, Mitamura T, Hosaka M, Wang L, Tanaka S and Sakuragi N: Clusterin is a potential molecular predictor for ovarian cancer patient's survival: targeting clusterin improves response to paclitaxel. J Exp Clin Cancer Res 30: 113, 2011.

22. Ferrara N, Hillan KJ and Novotny W: Bevacizumab (Avastin), a humanized anti-VEGF monoclonal antibody for cancer therapy. Biochem Biophys Res Commun 333: 328-335, 2005. 